\title{
The multidisciplinary approach to orthodontic-surgical protocols in the Maxillofacial Department of Pitié-Salpêtrière Hospital
}

\author{
A. Kerbrat ${ }^{1}$, J.B. Kerbrat ${ }^{2}$, A.S. Bourlon ${ }^{3}$, T. Schouman ${ }^{2,4}$, \\ P. Goudot ${ }^{2,4,5}$ \\ 1 Resident at the Hôpitaux de Paris \\ 2 Maxillofacial surgeon, Pitié-Salpêtrière \\ 3 Dental surgeon, Pitié-Salpêtrière \\ 4 Resident practitioner at the Hôpitaux de Paris \\ 5 University Professor
}

\section{ABSTRACT}

The creation of a multidisciplinary consultation in the maxillofacial service of the Pitié-Salpêtrière Charles-Foix hospital, improved the efficiency of patient care. They don't only see a maxillofacial surgeon and an orthondontist, they also have a follow up by an psychologist, a physiologist and a nutritionist. Preoperating day is the focal point of the patient care: it's the link between the preoperative time and the operative time.

\section{KEY WORDS}

Multidisciplinary, preoperative day, orthognatic surgery, orthodontics, physiotherapy, psychology

\section{INTRODUCTION}

Patient care is an intricate process that spans several years (fig. 1).

An orthodontic-surgical consultation at Pitié-Salpêtrière and Charles-Foix Hospital is a multidisciplinary consultation aimed at providing holistic care to patients who are candidates for orthodontic-surgical intervention. It is scheduled weekly and involves several specialists in each consultation: one or two surgeons, several orthodontists, a physiotherapist, and one or two psychologists.

Address for correspondence: 


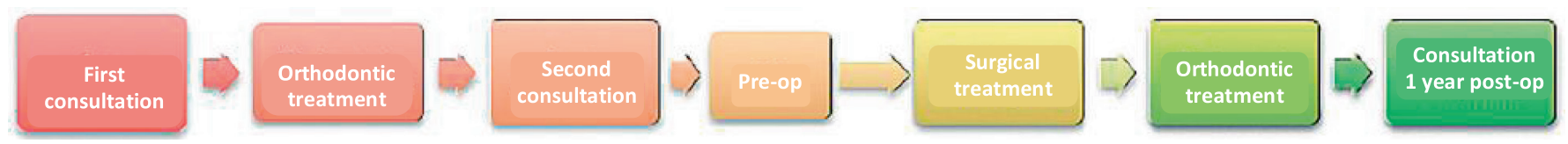

Figure 1

Organization of long-term patient care.

\section{THE FIRST CONSULTATION}

At the first consultation, patients meet the entire team for the first time. During this consultation, the diagnosis is made and a treatment plan is proposed. The various members of the surgical team are then introduced.

The patient's X-rays are examined (which include teleradiographs of the face and profile and a panoramic dental X-ray). A cephalometric study is conducted according to the methods proposed by Delaire and Sassouni. A treatment plan is then proposed to either conduct the surgery in one or two stages, for example, maxillary or mandibular extraction followed by a maxillomandibular osteotomy, which would usually be conducted after orthodontic prepa- ration or following the first surgery depending on the clinical situation.

In all scenarios, surgical risks are explained to patients who also receive an information pamphlet. They can therefore ask questions about the treatment phases, the surgery itself, the postoperative effects, and the orthodontic treatment, i.e., whether it is via vestibular or lingual multi-attachment or via an aligner. Informative videos reinforcing the information are available on the department's website: cmf-paris.com.

The psychologist's input during this consultation can address the possible psychological consequences of facial modifications beforehand and provide useful information regarding some psychiatric contraindications of orthodontic-surgical treatment.

\section{THE SECOND CONSULTATION}

Once the orthodontist deems the patient to be sufficiently prepared for surgery, the patient and his/her file (containing study models and recent X-rays, i.e., panoramic dental X-ray, teleradiographs of the face and profile with accompanying cephalometric analyses) are reviewed by the entire team.
Two dates are set: one for the operation and the other to conduct a series of necessary preoperative tests.

The patient is again informed of the associated risks, and the team ensures that the patient fully understands the written information received during the first consultation. 


\section{THE DAY BEFORE THE SURGERY}

One month before the surgery, all necessary appointments are grouped together on the same day to facilitate the following:

- Taking a new set of impressions with a facebow recorder. These impressions will allow the practitioner to report on the progress of orthodontic treatment undertaken and to identify the desired pre-and postoperative shifts.

- Consulting a physiotherapist. During this consultation the patient's swallowing, breathing, and posture are analyzed. The objective of this analysis is to look for disorders that can be corrected by physiotherapy sessions and by self-rehabilitation, thus improving the surgical outcomes $^{2,3}$.

- Appointment with the psychologist. Psychological care is essential as surgery inevitably affects the patient's face ${ }^{1}$. It is therefore important to ensure that the patient is aware of this change. During this appointment, the psychologist uses a questionnaire to detect potential psychiatric disorders that may contraindicate surgery. In addition, the psychologist analyzes the behavior of the patient's parents or spouse. This consultation is about $1 \mathrm{~h}$ in duration.
- A standardized photographic examination with photogrammetry [or three-dimensional (3D) photos] (fig. 2).

- Taking standard teleradiographs to perform a new cephalometric analysis in preparation for surgery.

- Scanning the facial mass from the vertex to the hyoid bone in millimeter sections to permit.

- visualization of the mandibular canal.

$-3 D$ simulation of surgery and the manufacture of custom-made models $^{4}$ (figs. 3a and b).

- Presurgical anesthesia consultation.

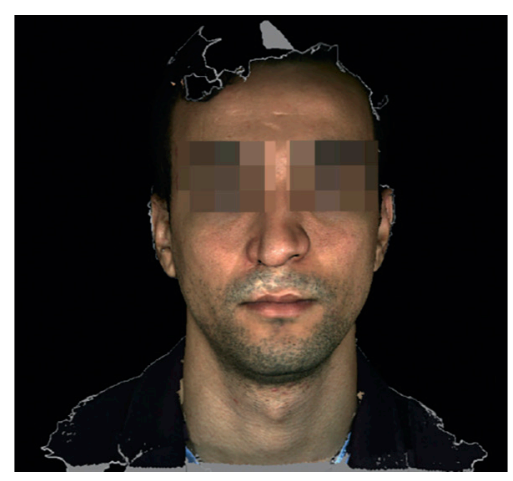

Figure 2

3D photograph of the patient. 


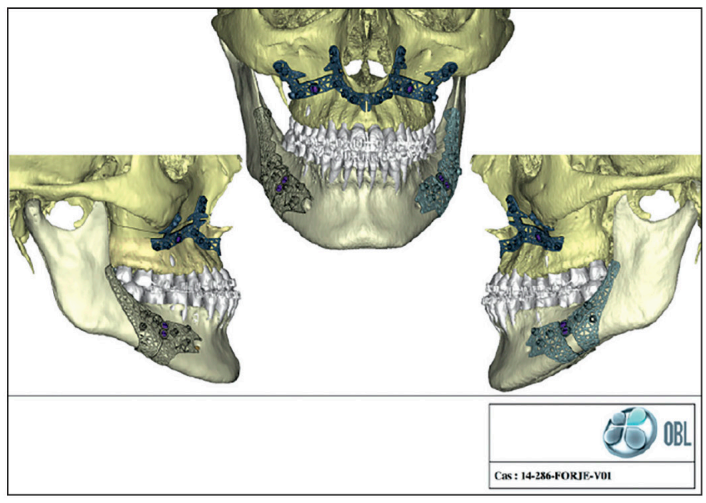

a)

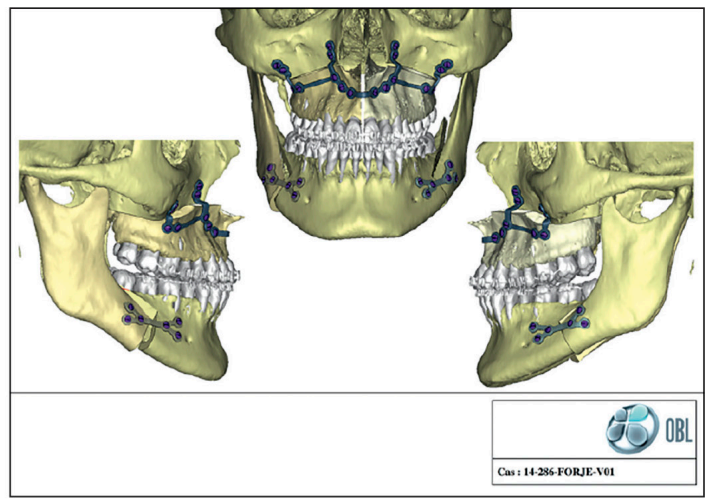

b)

Figure 3

a) Simulation of surgery with a cutting guide and pre drilling. b) Simulation of surgery with osteosynthesis plates.

The final stage of this day is the multidisciplinary orthodontic-surgical consultation. The resident maxillofacial surgeon presents the clinical case, the radiological assessment with the cephalometric studies, as well as the casts mounted on the articulator. The surgeon, orthodontist, physiotherapist, and psychologist discuss the procedure.

The therapist explains the need to commence rehabilitation immediately after surgery. To achieve this, a physical therapy prescription is prepared so that the patient is aware of the appointment dates (fig. 4).

During this consultation, one last appointment is planned to place surgical arches that will be attached to each interdental papilla.

This final stage allows the patient to receive a recap of pertinent information, to ask all useful questions, and to sign the consent form for the surgery.

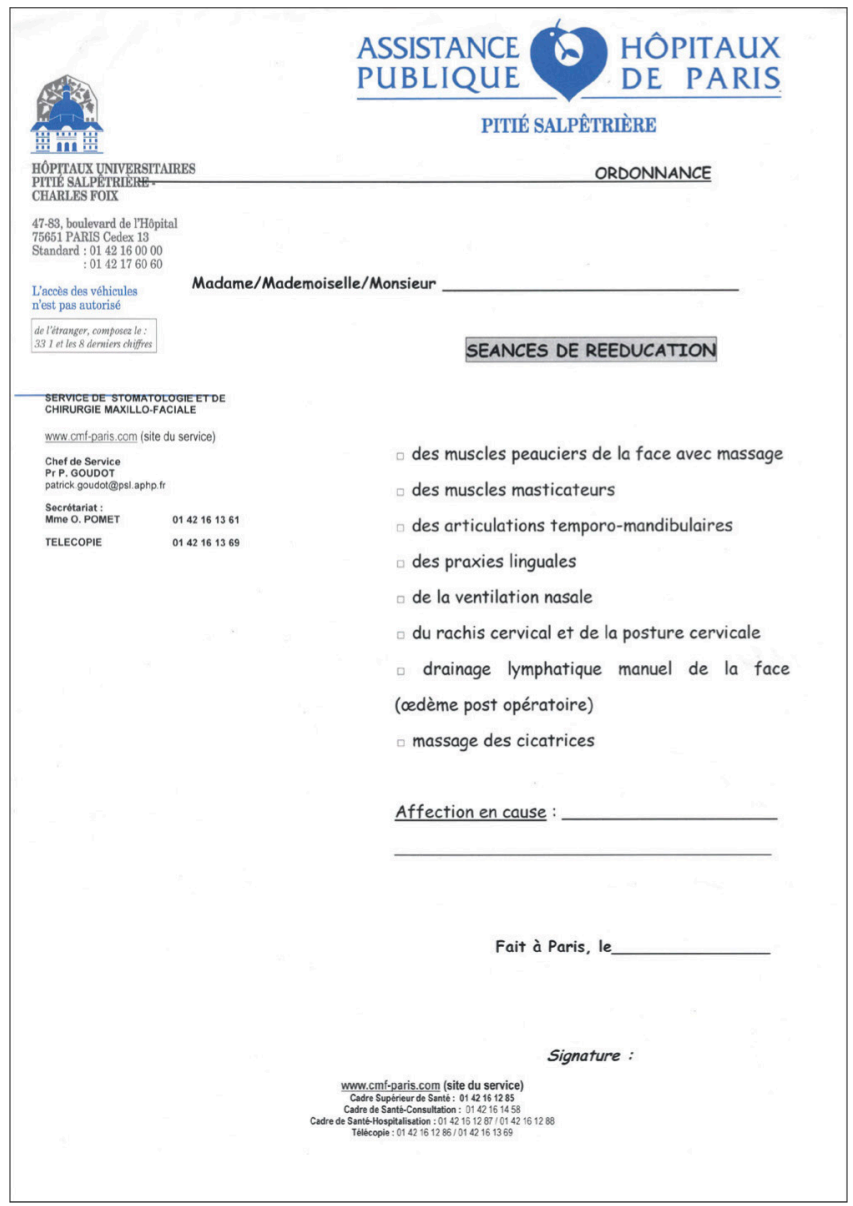

Figure 4

Example of a physical therapy prescription. 


\section{SURGERY AND CARE DURING HOSPITALIZATION}

The patient is hospitalized after undergoing conventional surgery. The duration of hospitalization varies and is generally 2 to 4 days.

Three surgical techniques are most often used in orthodontic surgery: mandibular sagittal surgery, LeFort 1 maxillary osteotomy, and genioplasty. The decision on the type of surgery to be performed is taken during the first appointment and is then confirmed on the day before surgery. The procedure is simulated in all three dimensions of space. The sites to be cut and drilled are clearly marked and the osteosynthesis plates are made custom made to fit each patient.

After surgery, the goal is to manage the pain as well as possible to effectively administer local treatments and prevent the occurrence of early postoperative complications.

While hospitalized, the patient starts nutritional management. It helps the patient adapt the diet to the constraints of surgery. Adapting to the texture of food is necessary. The other goal of the dietitian is to account for the patient's nutritional needs.

The absence of maxillomandibular blockage makes it easier to adapt to changes in food texture; in the first few days, a liquid diet is provided; then, a semi-liquid diet, such as purée foods, is provided. Then, normal, but chopped or ground, foods and finally, normal foods 45 days after the surgery are provided.

During the interview with the patient, the dietician guides as the patient on how to adapt to new eating habits. The patient is given a brochure to be able to eat at home and to make his/her own meals.

Lastly, physiotherapy with facial drainage is started. It continues after the patient has been released by the private practitioners.

\section{POSTOPERATIVE CONSULTATIONS}

The first postoperative consultation takes place approximately 10 days later and is conducted by the orthodontic-surgical team. The following appointments are scheduled at 1 month, 3 months, and 6 months after surgery. The surgeon ensures that there are no complications and that the patient continues to follow the treatment plan.

After the intervention, orthodontic treatment is continued to finalize the result. A restraint device will be given to the patient as soon as the orthodontic material is removed to maintain this result.

A new consultation date is decided 1 year later, with the same complementary examinations as before the surgery, with the exception of computed tomography, to evaluate the results of the procedure. The multidisciplinary analysis of the results makes it possible to assess their quality and discuss possible shortcomings for the benefit of subsequent patients. 
A psychological study of the patient's emotional state is conducted. Secondary physical changes caused by the surgery can have considerable effects on the patient's emotions.
Annual monitoring of the patient by the surgeon is ideally continued for up to 5 years after the surgery to ensure the stability of the result.

\section{CONCLUSION}

The demands for orthodonticsurgical therapeutic protocols have led Pitié-Salpêtrière Hospital to establish an orthodontic-surgical consultation. It allows for the comprehensive and multidisciplinary care of patients throughout the surgical preparation and then in postoperative period. The cohesion of the team helps reassure patients throughout the process.

Conflict of Interest: The authors have declared that they do not have any conflict of interest.

\section{BIBLIOGRAPHY}

1. Palumbo B, Cassese R, Fusetti S, Tartaro GP. Psychological aspects of orthognathic treatment. Minerva Stomatol 2006;55:33-42.

2. Takatsuji $\mathrm{H}$, et al. Effects of orthognathic surgery on psychological status of patients with jaw deformities. Int J Oral Maxillofac Surg 2015;44:1125-1130.

3. Teng TT, Ko EW, Huang CS, Chen YR. The Effect of early physiotherapy on the recovery of mandibular function after orthognathic surgery for Class III correction: part I-jawmotion analysis. J Craniomaxillofac Surg 2015;43:131-7.

4. Terzic A, Schouman T, Scolozzi P. Accuracy of morphological simulation for orthognatic surgery. Assessment of a 3D image fusion software. Rev Stomatol Chir Maxillofac Chir Orale. 2013 Aug 6. 